\title{
Fellow Column: COVID-19 Clinical Quick Guide for the Neonatologist
}

Anna G. Smith, MD, Abhineet Monti Sharma, MD

Case:

A 30-year-old gravida three mother presents to triage at $326 / 7$ weeks gestational age after feeling a gush of clear fluid overnight. Her obstetrics team admits her for further monitoring and administration of betamethasone due to the risk of prematurity. On admission, her temperature is $98.7 \mathrm{~F}$, heart rate is $80-90$ (sinus), her respiratory rate is 25 , her blood pressure is $110 / 60$, and she is saturating $100 \%$ on room air. To date, her pregnancy has been uncomplicated, and she has no significant past medical history. Her serologies at the time of admission are normal except for a pending GBS swab and SARS-CoV-2 nasal-pharyngeal swab. She does not report drug, tobacco, or current alcohol use. She is a nurse in an adult intermediate care unit. Her previous pregnancies have been uncomplicated and resulted in the delivery of two healthy, term neonates. The following day, she reports contractions of increasing frequency. On her assessment, she is febrile to $102.3 \mathrm{~F}$ and saturating $89 \%$. She is tachycardic with a sinus heart rate of 100 and visibly diaphoretic. Her blood pressure is 130/80. Her SARS-CoV-2 is still pending. Three hours after onset of developing symptoms, she delivers a male infant with APGAR scores of 8-9, weighing 1800 grams, and an initial temperature of 99.7F. The infant maintains age-appropriate saturations initially but eventually placed on CPAP +5 for tachypnea and drifting saturations. Following delivery, he is admitted to the neonatal intensive care unit due to gestational age, weight, and need for respiratory support. An X-ray obtained on admission shows findings consistent with respiratory distress syndrome of the newborn. Four hours following the infant's admission, the mother's SARS-CoV-2 swab returns positive. She is admitted to the adult Intensive Care Unit due to worsening respiratory status.

\section{"Four hours following the infant's admission, the mother's SARS-CoV-2 swab returns positive. She is admitted to the adult Intensive Care Unit due to worsening respiratory status."}

\section{Background:}

Severe acute respiratory syndrome coronavirus (2) (SARS-CoV-2) is the virus that causes coronavirus disease 2019 (COVID-19). As of April 2, 2020, 1.7\% of infections were among children aged < 18 years. Within this pediatric category, $23 \%$ of affected children were below age 2.(1) Understanding of COVID-19, along with recommendations for neonatologists and their patients, is evolving. The following is a summary of the literature available during April 2020 on select topics.

Prenatal Counseling:
Pregnant women should follow the same recommendations as non-pregnant persons for avoiding exposure to SARS-CoV-2. Current literature suggests that pregnancy does not appear to increase susceptibility to infection or worsen the clinical course. In areas of high incidence, a high percentage of mothers are reported to be asymptomatic. $(2,3)$ Data from New York City showed that with universal screening, $15 \%$ of pregnant mothers tested positive (33/215). $87 \%$ of these women had no symptoms at the time of presentation (29/215). In some centers, $33 \%$ of women were found to be positive during admission due to symptoms or implementation of universal screening. Among these women, $71 \%$ developed symptoms during their hospital stay. $(2,3)$ As a provider, consider telemedicine via phone or video for prenatal counseling services in order to minimize the risk of exposure. (4)

\section{Delivery Considerations:}

To reduce the risk of exposure, consider minimizing the number of providers only to include essential staff for delivery. In institutions without universal screening, keep in mind that the second stage of labor is considered an aerosol-generating procedure, which may require additional personal protective equipment (PPE). (5) Currently, there are no recommendations regarding cesarean section or vaginal delivery for SARS-CoV-2 positive mothers. $(4,6)$

\section{Resuscitation Considerations:}

For newborns who require resuscitation, the standard Neonatal Resuscitation Program (NRP) pathways can be followed. Intubation and airway considerations include the use of viral and bacterial filters on ventilator circuits to decrease aerosolization. (7) At this time, consider $\mathrm{N}-95$ or equivalent protection for all vaginal and cesarean section deliveries for Person of Interest (PUI) and known positive mothers.

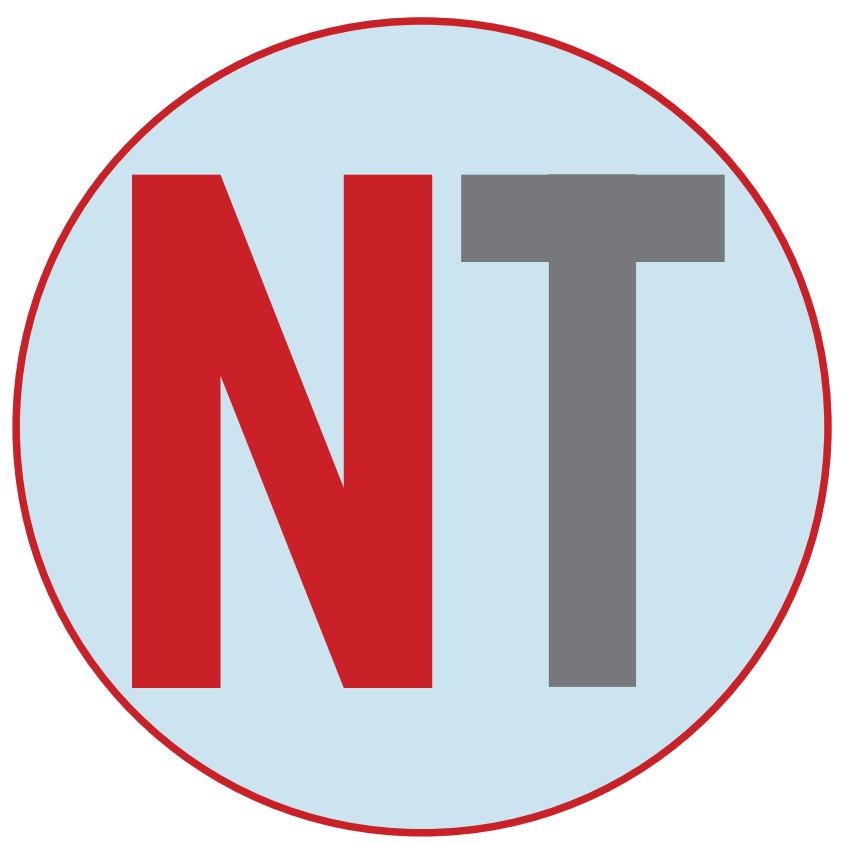




\section{Transmission and Newborn Testing:}

There is limited evidence of vertical transmission to neonates. Current studies cite a range from $11 \%(3 / 33)$ in the most extensive retrospective study to $14 \%$ (1/7) in another retrospective study.(8) 9 There is increasing evidence that neonates are positive for lgM and IgG suggesting that antibodies either pass from the mother (IgG) or are produced by the infant (IgM). (10) A review of bodily fluids, including amniotic fluid, cord blood, neonate nasopharyngeal swab, and breast milk, have all tested negative to date. (11) Newborns can still acquire COVID-19 through close contact with infected mothers as SARS-CoV-2 main routes of transmission are respiratory droplets, via contact and aerosol, and contaminated objects like toys and baby bottles. (12) At this time, infants born to SARS-CoV-2 positive mothers should be considered PUIs initially, and appropriate PPE should be used. Current recommendations suggest testing vertically exposed newborns at 24 hours of life to increase the likelihood of capturing replicating viral RNA as opposed to contaminating RNA and repeat testing at 48 hours of life. (6) If the infant is determined to be negative on both tests, no additional PPE is required. Labs of neonates who test positive for SARS-CoV-2 are nonspecific. Positive neonates are more likely to have elevated WBC compared to negative neonates. (10) No lymphopenia has been noted in neonates, as compared to literature cited for older children and adults. A review of computed tomography and X-ray findings among infected neonates has demonstrated no specific findings. $(10,13)$

\section{"Current recommendations suggest} testing vertically exposed newborns at 24 hours of life to increase the likelihood of capturing replicating viral RNA as opposed to contaminating RNA and repeat testing at $\mathbf{4 8}$ hours of life. (6)"

\section{Outcomes in Neonates:}

To date, there is a relatively low incidence of poor outcomes in neonates. (14) Primary clinical issues reported in the literature are related to postmenstrual age (PMA) at birth as opposed to symptoms of SARS-CoV-2. There is a higher instance of preterm births and preterm labor in patients who test positive for COVID-19, but this seems to be related to maternal factors as opposed to neonatal factors. Current data suggests that $14 \%$ of newborns born to SARS-CoV-2 positive mothers were born before 36 weeks' gestation. (14) The youngest positive infant described in the literature was born at 31 weeks PMA and had complications related to prematurity. (8) The average APGAR scores cited in present literature for these newborns have been $8-9$. $(10,14)$ Clinical features of symptomatic newborns born to mothers with confirmed COVID-19 may include fever, pneumonia, respiratory distress syndrome, cyanosis, lethargy, feeding intolerance, emesis, diarrhea, and abdominal distention. Sepsis, DIC, and death have been rare complications cited in a single case report. $(10,14,15)$ The youngest infant born to a SARS-CoV-2 mother who died was born at 34 weeks' gestation and died at 8 days of life. The patient's demise was due to multisystem organ failure without a clear etiology. The infant was found to be PCR negative on multiple tests throughout the hospitalization. (14) The average length of NICU stay has not been found to vary from the average length of stay for neonates who are otherwise well. $(8,10)$ Postnatal management of neonates born to SARS-CoV-2 positive mothers depends on the institution. The variation highlights the importance of identifying a second caregiver in the event of worsening maternal illness.

\section{Routine Care:}

Current recommendations for routine newborn care in the setting of SARS-CoV-2 positive testing for mother and/or baby are based on evolving expert guidelines. $(4,6)$ Infants should be bathed as soon as possible to facilitate the removal of viral particles. Mothers should be encouraged to pump early to establish supply. Currently, no data suggesting that SARS-CoV-2 is present in breastmilk exists. (11) Thus, mothers should pump and feed, if possible. Breastmilk may contain protective immunologic factors against SARS-CoV-2. If it is not feasible to separate mother and infant or if the mother chooses to room with her infant, it is recommended that she maintain six feet distance unless caring for her infant. Well appearing newborns should receive all indicated care, including timely administration of vaccines and circumcision. At discharge, it is recommended to maintain separation of mother and infant for at least 14 days if the mother is positive and the baby is negative. In such cases, the infant should be discharged home with a caregiver who has tested negative, and the mother should maintain distance when possible. At a minimum, a mother should use hand hygiene and wear a face mask until she is free of fevers for 72 hours, remains asymptomatic for at least seven days, or she has a negative repeat SARS-CoV-2 test. Positive infants may be discharged with close follow up through the first 14 days. Methods of follow up include telemedicine or in-office visits with the pediatrician. All caregivers should follow proper hand hygiene and wear appropriate PPE.

\section{"Positive infants may be discharged with close follow up through the first 14 days. Methods of follow up include telemedicine or in-office visits with the pediatrician. All caregivers should follow proper hand hygiene and wear appropriate PPE."}

\section{Case Continued:}

Given the maternal fever and neonatal requirement for respiratory support, blood cultures were obtained, and the infant was started on antibiotics. The infant did not develop an oxygen requirement and was weaned from respiratory support the following day. $\mathrm{Na}-$ sopharyngeal swabs for SARS-CoV-2 from the infant remained negative at 24 and 48 hours of life screens. Antibiotics were discontinued at 48 hours, and PPE precautions were removed at the same time. The infant required nasogastric gavage feeds and remained in the NICU until 36 weeks PMA. He was discharged home with his grandmother and father while his mother remained in the hospital. She was discharged a month after delivery. At the time of discharge, her SARS-CoV-2 swabs were negative. She was otherwise asymptomatic at the time of discharge.

\section{References}

1. 1- CDC COVID-19 Response Team. Coronavirus Disease 
2019 in Children - United States, February 12-April 2, 2020. MMWR Morb Mortal Wkly Rep. 2020; 69 (14):422. Epub 2020 April 10

2. Breslin, N. B.-B. (2020). COVID-19 infection among asymptomatic and symptomatic pregnant women: Two weeks of confirmed presentations to an affiliated pair of New York City hospitals. American Journal of Obstetrics \& Gynecology MFM, 100118.

3. Sutton, D. K. (2020). Universal Screening for SARS-CoV-2 in Women Admitted for Delivery. New England Journal of Medicine.

4. Chandrasekharan, P. V.-C.-2. (2020). Neonatal Resuscitation and Postresuscitation Care of Infants Born to Mothers with Suspected or Confirmed SARS-CoV-2 Infection. American Journal of Perinatology.

5. Tran, K. C.-S. (2012). Aerosol generating procedures and risk of transmission of acute respiratory infections to healthcare workers: a systematic review. PloS one.

6. Puopolo, K. M. (2020). Management of Infants Born to Mothers with COVID-19. American Academy of Pediatrics Committee on Fetus and Newborn, Section on Neonatal-Perinatal Medicine, and Committee on Infectious Diseases.

7. Dubler, S. Z. (2016). Bacterial and viral contamination of breathing circuits after extended use - an aspect of patient safety? Anaesthesiologica Scandinavica.

8. Zeng, H. X. (2020). Antibodies in infants born to mothers with COVID-19 pneumonia. JAMA.

9. Yu, N. L. (2020). Clinical features and obstetric and neonatal outcomes of pregnant patients with COVID-19 in Wuhan, China: a retrospective, single-centre, descriptive study. The Lancet Infectious Diseases.

10. Zeng, L. X. (2020). Neonatal early-onset infection with SARS-CoV-2 in 33 neonates born to mothers with COVID-19 in Wuhan, China. JAMA pediatrics.

11. Chen H, G. J. (2020). Clinical characteristics and intrauterine vertical transmission potential of COVID-19 infection in nine pregnant women: a retrospective review of medical records. Lancet.

12. Cao, Q., Chen, Y. C., Chen, C. L., \& Chiu, C. H. (2020). SARS-CoV-2 infection in children: Transmission dynamics and clinical characteristics. J Formos Med Assoc, 119(3), 670-673.

13. Xia, W. S. (2020). Clinical and CT features in pediatric patients with COVID-19 infection: Different points from adults. Clinical Pathology.

14. Zhu, H. W. (2020). Clinical analysis of 10 neonates born to mothers with 2019-nCoV pneumonia. Translational Pediatrics, 51.

15. Wang, L., Shi, Y., Xiao, T., Fu, J., Feng, X., Mu, D., ... \& Lu, G. (2020). Chinese expert consensus on the perinatal and neonatal management for the prevention and control of the 2019 novel coronavirus infection. Annals of Translational Medicine, 8(3).

Disclosure: The authors have no disclosures
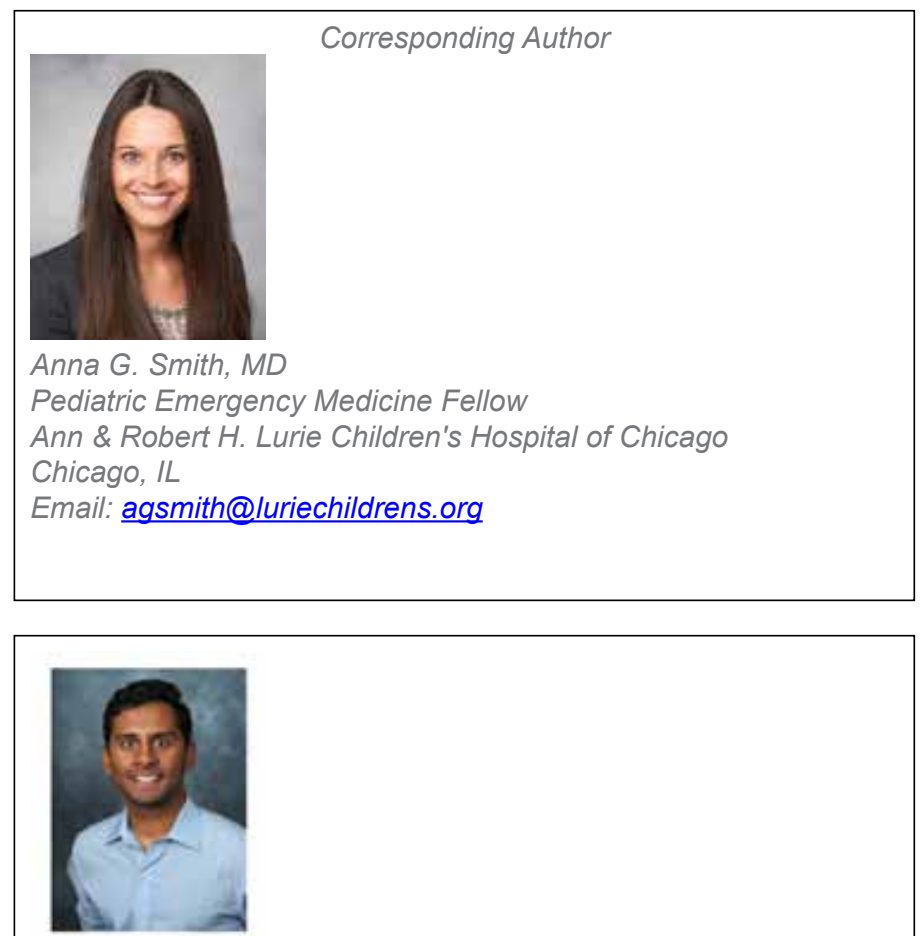

Abhineet Monti Sharma, MD

Neonatology Fellow

Ann \& Robert H. Lurie Children's Hospital of Chicago Chicago, IL

Email: abhineet.sharma@northwestern.edu

Fellow's Column is published monthly.

- Submission guidelines for "Fellow's Column":

- 2000 word limit not including references or title page. Ex ceptions will be made on a case by case basis

- $\quad$ QI/QA work, case studies, or a poster from a scientific meeting may be submitted..

- Submission should be from a resident, fellow, or NNP in training.

- Topics may include Perinatology, Neonatology, and Younger Pediatric patients.

- No more than 20 references.

- Please send your submissions to:

Elba Fayard, MD

Interim Fellowship Column Editor LomaLindaPublishingCompany@gmail.com

NEONATOLOGY TODAY is interested in publishing manuscripts from Neonatologists, Fellows, NNPs and those involved in caring for neonates on case studies, research results, hospital news, meeting announcements, and other pertinent topics.

Please submit your manuscript to: LomaLindaPublishingCompany@gmail.com 